\title{
EELS and EFTEM Analysis of Biological Materials
}

\author{
R.D. Leapman and M.A. Aronova \\ Laboratory of Cellular Imaging \& Macromolecular Biophysics, NIBIB, NIH, Bethesda, MD 20892
}

The high sensitivity of electron energy-loss spectroscopy (EELS) for detecting light elements at the nanoscale in the analytical electron microscope makes it a valuable technique for applications to biological materials [1]. In particular, EELS provides quantitative information about elemental distributions within subcellular compartments, and the number of specific atoms that are bound to individual macromolecular assemblies. EELS data can be acquired either in the fixed beam energyfiltered transmission electron microscope (EFTEM) or in the scanning transmission electron microscope (STEM), and recent progress in the development of both approaches has greatly expanded the range of applications for EELS analysis. Near single atom sensitivity is now achievable for certain elements bound to isolated macromolecules [2], and it has become possible to obtain three-dimensional compositional distributions from sectioned cells through EFTEM tomography [3].

Fig. 1 illustrates the complementarity and different operating regimes for data acquisition [1]. STEMEELS (regime A) typically provides image sizes of around $10^{4}$ pixels, whereas EFTEM (regime B) can provide image sizes of $\sim 10^{6}$ pixels, depending on the binning factor of the CCD camera. For 3-nm pixels, these image sizes correspond to fields of view of $3 \mu \mathrm{m}$ for EFTEM but only $0.3 \mu \mathrm{m}$ for STEMEELS. New developments in detector technology (regime $C$ in Fig. 1) now provide much faster read-out times and the possibility of STEM-EELS spectrum images that contain more than $10^{5}$ pixels.

To detect low elemental concentrations, it is often preferable to focus nanoampere currents into probe diameters of a few nanometers, and to utilize the high efficiency of STEM-EELS hyperspectral imaging. For example, such an approach has been used to map distributions of single molecules of ferritin, an important iron storage protein, in 100-nm sized sub-regions of sectioned brain tissue, as shown in Fig. 1; quantitative analysis of such images can be related to magnetic contrast in magnetic resonance imaging [4]. STEM-EELS is also the technique of choice when beam damage is a limiting factor because the entire spectrum is recorded in parallel at each pixel.

In EFTEM, spectral information is acquired serially so this approach is much less efficient than STEMEELS. However, when there is a need to study compositional variations over cellular regions that are several micrometers across, the EFTEM spectrum-imaging approach can be advantageous because it enables acquisition of elemental maps containing as many as $10^{6}$ pixels in a few minutes if concentrations are high enough. For example, EFTEM can be used to map distributions phosphorus, which is an intrinsic marker for nucleic acids [5], and distributions of proteins that are rich in sulfurcontaining amino acids, such as insulin secreting granules in beta cells of pancreatic islet of Langerhans [6,7], as shown in Fig. 1.

EFTEM and EELS are also valuable for characterizing multi-component bionanoparticles. These include nanocomplexes of organic or polymer phases containing metals used as magnetic resonance contrast agents [8], and mixed organic and inorganic nanoparticles used as optical probes [9,10]. 


\section{References}

[1] M.A. Aronova, R.D. Leapman, MRS Bulletin 37 (2012) 53.

[2] R.D. Leapman, J. Microsc. 210 (2003) 5.

[3] M.A. Aronova et al., J. Struct. Biol. 160 (2007) 35.

[4] M. Fukunaga et al., Proc. Natl. Acad. Sci. USA 107(2010) 3834.

[5] D.P. Bazett-Jones, M.J. Hendzel , M.J. Kruhlak, Micron 30 (1999) 151.

[6] G. Goping et al., Microsc. Res. Tech. 61 (2003) 448.

[7] T. Cai et al., Diabetologia 54 (2011) 2347.

[8] A.A. Sousa et al., Nanomedicine 4 (2009) 391.

[9] J. Xie et al., ACS Nano 5 (2011) 3043.

[10] This research was supported by the intramural program of the National Institute of Biomedical Imaging and Bioengineering, National Institutes of Health.
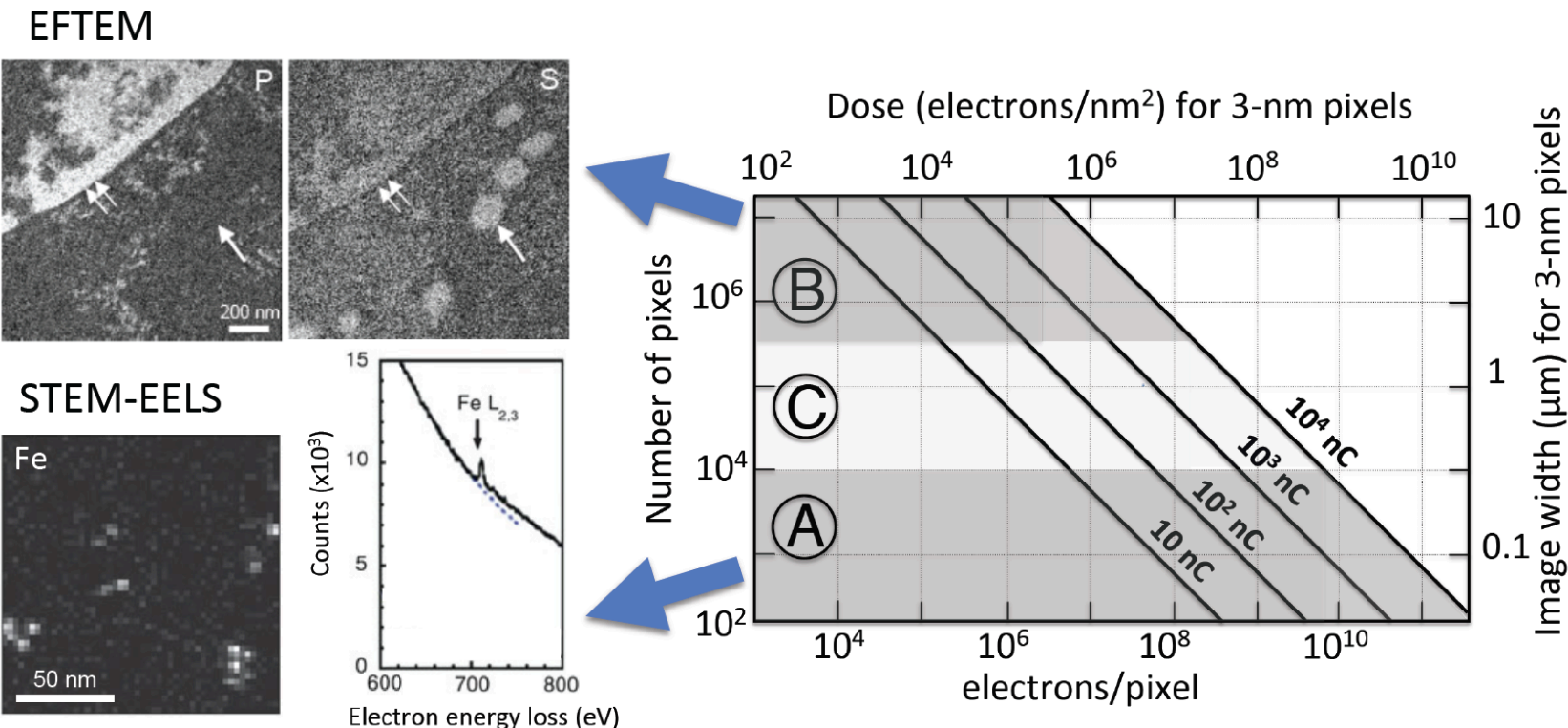

Fig. 1. Diagram showing different operating conditions (numbers of pixels, electron dose, field of view) for acquiring compositional maps of biological materials by STEM-EELS and EFTEM; diagonal lines indicate the total number of electrons (nano-coulombs) incident on the specimen area during course of acquisition. In regime (A), the spectrometer/imaging filter (e.g., Gatan Tridiem GIF) is used to acquire 1024-channel EELS data at each pixel in STEM mode to give a spectrumimage, whose number of pixels is typically limited by the available probe current and minimum read-out time of the detector; this is illustrated by STEM-EELS mapping of iron in unstained cerebral cortex, which reveals the distribution of ferritin molecules, as is evident by the EELS Fe $\mathrm{L}_{2,3}$ edge extracted from a single ferritin core [4]. In regime (B), the imaging filter (e.g., Gatan Tridiem GIF) is used to acquire a series of EFTEM images, containing of the order of $10^{6}$ pixels, from which elemental distributions can be determined; this is illustrated by an EFTEM spectrumimage from an insulin-secreting beta cell in a thin section of mouse islet of Langerhans, which reveals the phosphorus distribution ( $\mathrm{P}_{2,3}$ edge) in nuclear chromatin (double arrows) and cytoplasmic ribosomes, and sulfur-rich insulin granules ( $\mathrm{S} \mathrm{L}_{2,3}$ edge) (single arrow) [6,7]. Regime (C) represents the newest generation of imaging filter (e.g., Gatan Quantum GIF), which can read out spectra in $\sim 1 \mathrm{~ms}$, enabling much larger STEM-EELS spectrum-images containing $>10^{5}$ pixels. 